\title{
Enquête
}

Archives de la revue Enquête

5 | 1997

Débats et controverses

\section{Le linguistic turn en Angleterre}

Notes sur un débat et ses censures

The linguistic turn in England

\section{Simona Cerutti}

Traducteur : Jean Boutier

\section{(2) OpenEdition}

Journals

Édition électronique

URL : http://journals.openedition.org/enquete/1183

DOI : 10.4000/enquete.1183

ISSN : 1953-809X

Éditeur :

Cercom, Éditions Parenthèses

Édition imprimée

Date de publication : 1 septembre 1997

Pagination : 125-140

Référence électronique

Simona Cerutti, «Le linguistic turn en Angleterre », Enquête [En ligne], 5 | 1997, mis en ligne le 15 juillet 2013, consulté le 20 mars 2020. URL : http://journals.openedition.org/enquete/1183 ; DOI : https:// doi.org/10.4000/enquete. 1183

Ce document a été généré automatiquement le 20 mars 2020 


\title{
Le linguistic turn en Angleterre
}

\author{
Notes sur un débat et ses censures \\ The linguistic turn in England
}

\section{Simona Cerutti}

Traduction : Jean Boutier

[1] Je m'efforcerai dans ces pages de reconstruire les formes prises par un débat historique en un lieu spécifique. Le débat concerne le linguistic turn, c'est-à-dire, en bref, ce courant qui assigne aux langages un rôle décisif dans la construction de la réalité sociale, en particulier des mouvements collectifs. Le lieu est l'une des plus prestigieuses revues d'histoire sociale anglaise, Social History. Le choix de limiter le terrain d'enquête à cette seule revue (avec, au maximum, quelques incursions dans des revues « jumelées » comme History Workshop Journal) a deux significations. La première est banale : elle sert à délimiter un objet qui, tout comme la discussion autour du linguistic turn, a pris des dimensions énormes (surtout dans la culture anglo-saxonne). La seconde est plus fondée: la série d'articles parus dans Social History constitue désormais un corpus cohérent, qui se présente comme un entrecroisement serré de dialogues - attaques et ripostes entre un nombre restreint d'individus - et exige une lecture cumulative ${ }^{1}$. Le ton particulièrement violent et apocalyptique pris par ce débat est un autre élément de spécificité, qu'il faut expliquer. Que se joue-t-il autour du linguistic turn en Angleterre? Que signifie la menace de la "fin de l'histoire sociale", qui revient en plus d'un article, et pourquoi cette délégitimation institutionnelle, qui impliquerait l'existence même de la revue-hôte, s'accompagne-t-elle d'accusations personnelles de trahison, ou de mauvaise foi, etc. ? Cherchons les premiers éléments de compréhension dans une simple reconstruction chronologique du débat.

2 [2] L'article inaugural est en fait l'œuvre de deux historiens américains. Il s'agit d'une réflexion approfondie sur un livre important, Languages of Class, dont l'auteur, Gareth Stedman Jones, est l'un des plus prestigieux historiens des classes travailleuses de l'Angleterre du $\mathrm{XIX}^{e}$ siècle $^{2}$. Le livre, c'est bien connu, a représenté un tournant fondamental dans l'étude des mouvements politiques par rapport à la tradition historiographique incarnée par-dessus tout par Edward P. Thompson, le maître de Jones (et de bon nombre des participants du débat). Résumons-en la genèse et les 
arguments, tels qu'ils nous sont présentés par l'auteur dans son introduction. Languages of Class est un recueil d'articles, dont certains, déjà publiés, ont été partiellement modifiés. Ils témoignent d'un parcours intellectuel qui conduit de Outcast London, un premier livre sur les travailleurs occasionnels à Londres entre $\mathrm{XIX}^{\mathrm{e}}$ et $\mathrm{Xx}^{\mathrm{e}}$ siècle $^{3}$, à un tournant théorique fondamental, le linguistic turn.

Outcast London se plaçait dans un rapport de continuité explicite vis-à-vis du travail de Thompson sur la formation de la classe ouvrière anglaise ${ }^{4}$; il en acceptait les présupposés et les méthodes (en particulier l'articulation entre agency et structure qui, pour Thompson, était à l'origine de la formation d'une conscience de classe) et se proposait simplement d'étendre l'enquête dans le temps. Mais les choses changent lorsque Stedman Jones se pose le problème d'analyser de plus près, et de façon plus explicite, le thème, central pour les historiens des mouvements politiques anglais, que constitue la fin du chartisme. Ce qui l'amène à poser plus explicitement l'articulation entre culture et politique. Deux articles du recueil témoignent de cet effort (« Workingclass culture and working-class politics in London, 1870-1900»; « Why is the Labour Party a mess?»); mais c'est avec «Rethinking Chartism $^{5}$ » que survient le tournant théorique.

4 Les explications de la défaite du chartisme, écrit Stedman Jones, étaient en même temps trop vagues et trop économistes : elles évoquaient la défaite d'une Weltanshaung d'artisans, face à l'avancée d'une économie de marché en substance capitaliste. L'interrogation plus approfondie sur ce que peut être une conscience de classe (et comment elle peut disparaitre) porte Stedman Jones à la conviction de devoir renverser ses propres catégories interprétatives: il est nécessaire d'abandonner la catégorie d'idéologie, utilisée habituellement pour relier un ensemble de croyances et de convictions à la condition matérielle objective d'un groupe social. «Arrivé à ce point, poursuit Stedman Jones, je décidai de renverser mes présupposés initiaux : étant donné l'existence d'une base fondée de mécontentement matériel, ce n'était pas la conscience (ou l'idéologie) qui produisait la politique, mais la politique qui produisait la conscience. " Le déplacement de l'approche par rapport aux études antérieures sur le chartisme est radical. Il est nécessaire d'analyser les revendications du mouvement non comme des miroirs fidèles ou des traductions directes des conditions matérielles des travailleurs, mais plutôt comme des éléments d'organisation de ces expériences matérielles. «Le problème était théorique. Il concernait le rôle à assigner au langage, à la conscience et à l'expérience ", l'abandon des explications causales simplifiées et la recherche nouvelle des liens possibles entre ces termes. En particulier, le statut du langage exigeait d'être reconsidéré: non pas un médium entre l'expérience et ses expressions, mais un objet doté d'une matérialité propre. La référence à Saussure est évidente et explicite: elle sert surtout à revendiquer «l'impossibilité d'abstraire l'expérience du langage qui en structure les articulations ${ }^{6} »$. Mais elle sert aussi, de façon plus radicale, à affirmer le caractère non directement référentiel du langage, dont on nie la possibilité d'être ramené à une réalité sous-jacente. Stedman Jones propose donc une nouvelle donne, et révise en profondeur les modèles de causalité linéaire (condition matérielle/production de conscience/revendications), largement adoptés par l'historiographie du mouvement ouvrier.

5 Ce programme de révision théorique a d'importantes conséquences du point de vue des opérations: comprendre le chartisme signifie maintenant pour l'essentiel en reconstruire les expressions, et donc « explorer la relation systématique entre termes 
et propositions à l'intérieur du langage, plutôt que mettre des propositions particulières en relation directe avec une supposée existence réelle dont elles seraient l'expression "; « nous ne pouvons pas décoder le langage politique pour atteindre une expression primitive et matérielle des intérêts, du moment que c'est la structure discursive du langage politique qui engendre et définit les intérêts. Nous devons au contraire étudier la production des intérêts, identifications, revendications et aspirations à l'intérieur de ces mêmes langages politiques ${ }^{7}$ ». Ce qui est revendiqué, c'est le caractère " préfiguratif » et non réflexif du langage, et donc aussi son caractère relationnel (il existe un rapport direct entre les énoncés et le public auquel ils s'adressent). S'il est évident que le but officiel du discours politique est de fournir une réponse aux besoins et aux questions préexistantes, dans les faits, le but essentiel est de créer et de diriger besoins et questions, de modifier les comportements des individus auxquels le langage s'adresse.

6 Cohérent avec le programme ainsi énoncé, «Rethinking Chartism » est une analyse des revendications, des arguments et du langage politique utilisés dans les documents produits par les chartistes eux-mêmes. Il aboutit à constater une discontinuité entre revendications et conditions matérielles vécues par les classes travailleuses (le langage de l'exclusion politique et de la corruption était beaucoup plus ancien que les nouvelles conditions de travail, ses origines étant à rechercher dans une tradition radicale qui remonte au moins au XVII ${ }^{\mathrm{e}}$ siècle).

7 Peu après sa publication, Languages of Class suscite quelques comptes rendus critiques ${ }^{8}$; mais ce n'est pas un hasard si le débat dont nous nous occupons ici n'éclate que beaucoup plus tard, peu après la parution d'un livre qui renvoie explicitement aux réflexions de Stedman Jones, Visions of the People. Industrial England and the question of class, 1848-1914 (Cambridge University Press, 1991), dont l'auteur est Patrick Joyce. Le livre part d'une critique des notions mêmes de classe, de conscience et d'expérience, telles qu'elles ont été comprises par l'historiographie marxiste (et par Thompson en particulier), et se propose de leur opposer l'analyse des valeurs que les classes travailleuses victoriennes exprimaient réellement. Dans ce but, Joyce travaille sur trois domaines, la politique, le travail et la culture, analysant tour à tour les discours et les langages qui les dominent. Il découvre ainsi que la notion de classe est extraordinairement rare, alors qu'un langage populiste domine tous les discours. Le protagoniste de la société victorienne, c'est le peuple - vertueux ou besogneux, etc. - et non la classe. Par ses méthodes d'analyse plus encore que par ses résultats, Visions of the People est un livre radical. Le caractère non référentiel du langage y est affirmé beaucoup plus fermement que dans le livre de Stedman Jones, non seulement en théorie, mais à travers l'adoption d'une approche totalement dominée par les discours. Ce qui vaut aussi pour la confiance dans la capacité du langage à organiser l'expérience sociale.

8 [3] Le premier article de notre dossier, celui de D. Mayfield et S. Thorne, part en apparence du travail de Stedman Jones, mais l'essentiel des arguments les plus critiques concernent en réalité Visions of the People. Le problème examiné de la façon la plus systématique est celui du caractère autoréférentiel attribué au langage. Selon Mayfield et Thorne, c'est le fruit d'un malentendu ou, pire, d'une lecture infidèle de Saussure (sur l'autorité duquel l'argument était fondé).

L'approche sémiologique qui reconnaît dans le langage un système de signes (et pas seulement un système symbolique qui renverrait à des signifiés préexistants) ne nie pas que ceux-ci soient structurés en métaphores (signifiants) qui se réfèrent à 
quelque chose d'autre (signifié). La sémiologie met plutôt en discussion notre approche naïve, selon laquelle la différence entre objet désigné et instrument de désignation serait seulement une différence de catégories, entre un phénomène réel et un épiphénomène, une "représentation »... L'écart entre le signe et le signifié est au contraire interne au langage lui-même et en informe la structure : chaque acte discursif est structuré métaphoriquement comme l'identification d'une chose dans les termes de quelque chose de différent. Le lien entre signe et signifié est établi historiquement à travers le pouvoir « étranger» des conventions sociales. Et c'est pour cela que le rapport entre signe et signifié est discontinu'.

Tout comme chez Stedman Jones, le langage ne peut donc être pensé comme un événement secondaire, qui se superpose à une réalité pré-existante. Toutefois, le langage est référentiel. Les métaphores renvoient à quelque chose d'autre. Ce ne sont pas des verres transparents, ni même des instruments dotés du pouvoir autonome de créer à partir de rien des objets, des groupes, des intérêts, comme le prétendent Stedman Jones ou Joyce. Le devoir de l'historien est d'étudier le rapport complexe et discontinu entre des objets et leurs représentations, non celui de nier l'existence du social ou d'unifier les deux moments. Ce faisant, Stedman Jones et Joyce n'ont fait que reproduire, en l'inversant, la dichotomie entre social et politique, trop souvent proposée par la vieille "nouvelle histoire sociale». Les raisons de l'adhésion au chartisme ou celle de sa défaite restent obscures, une fois que l'autonomie du social a été simplement substituée à celle du politique.

Des thèmes analogues sont repris dans l'intervention suivante, celle de J. Lawrence et M. Taylor, qui se situent toutefois de façon polémique vis-à-vis de Mayfield et Thorne. Les reproches qu'ils leur adressent concernent plutôt les modes que les contenus du débat: un ton dogmatique et normatif; une politisation inopportune (Mayfield et Thorne avaient suggéré que le problème, non résolu et très actuel, du thatchérisme populaire pouvait être à l'origine d'élaborations théoriques qui, même pour le passé, justifiaient la scission entre social et politique); le renvoi implicite à des orthodoxies qui tendent à délégitimer tout parcours "nouveau ", etc. En bref, on assisterait à la défense de la bonne vieille histoire sociale thompsonienne contre les nouveaux méchants maîtres. Ce ton serait d'autant plus inopportun, prétendent Lawrence et Taylor, que la réflexion sur le rôle du langage se situerait dans la stricte continuité de la tradition de l'histoire sociale anglaise. On évoque à ce propos les réflexions de Thompson lui-même, ou l'éditorial du numéro de History Workshop Journal, « Language and History ", daté de 1980 et signé par M. Ignatieff, R. Samuel et G. Stedman Jones. D'où non pas rupture mais continuité. Le problème est tout autre que formel. La discussion autour du ton, des orthodoxies, des fractures est un caractère distinctif de ce débat, capable d'en expliquer l'enjeu. Nous allons y revenir.

Lawrence et Taylor ont l'énorme mérite d'apporter une clarification essentielle : mettre en lumière la grande distance qui sépare la recherche et les réflexions théoriques de Stedman Jones de celle de P. Joyce, qui ont été au contraire imprudemment confondues par Mayfield et Thorne. L'interrogation de Stedman Jones, et son approche de la dimension linguistique - soutiennent Lawrence et Taylor -, témoignent de sa recherche constante pour analyser les articulations entre sphère sociale et sphère politique, alors que Joyce "n'est pas loin de nier l'existence de tout rapport» entre ces deux dimensions ${ }^{10}$. La distinction est essentielle, et saute en effet aux yeux de quiconque analyse Languages of Class et Visions of the People ${ }^{11}$; elle sera utilement reprise au cours $\mathrm{du}$ débat, par exemple par $\mathrm{G}$. Eley et $\mathrm{K}$. Nield, et est à la base de la très lucide réflexion d'un historien anglais, Miles Taylor, qui est intervenu récemment sur ces questions ${ }^{12}$. 
Le second reproche adressé à Mayfield et Thorne n'est pas moins utile : ils ont traité Languages of Class comme un manifeste idéologique, sans examiner tous les problèmes « historiques et empiriques » que le livre soulève. Lawrence et Taylor insistent sur cette dimension empirique, qui les conduit à discuter des résultats de l'analyse de Stedman Jones et de l'usage qu'il fait des sources à sa disposition. D'où une critique sévère des contenus du travail et de ses conclusions: Languages of Class "accentue le pouvoir créatif et novateur des discours politiques formels, au détriment des rapports complexes entre les langages politiques et les discours informels à travers lesquels les gens interprètent leur propre vie et le monde qui les entoure ${ }^{13}$ ». L'utilisation prédominante des sources politique officielles a en fait lourdement orienté les résultats de la recherche; tout comme l'abandon total de toute source non discursive. La renonciation à toute analyse sociale - soutiennent Lawrence et Taylor - n'est pas une bonne stratégie à suivre pour qui, non satisfait par les approches essentialistes, veut s'interroger sur la complexité des rapports entre conditions matérielles et mobilisation politique, sur les notions de groupe, d'expérience, d'intérêts. Le croisement des sources semble au contraire à Lawrence et Taylor une voie féconde, du moment qu'elle peut permettre de s'interroger, par exemple, sur l'hétérogénéité des groupes sociaux, qu'elle peut rendre moins déterministe la notion d'intérêt, qu'elle peut dénaturaliser l'expérience que les acteurs concrets peuvent avoir vécue au cours de leur vie. L'impasse de la vieille histoire sociale vient de la rigidité de ses catégories d'analyses et de leur extériorité par rapport aux objets (elles servent plus à définir qu'à analyser). Une réflexion sur les sources et sur la façon de les lire devrait permettre d'en sortir.

[4] Conservons à l'esprit ces arguments : ils ne seront repris en cours de ce débat que pour lui nier toute légitimité. La référence à l'empirisme qui suscite les protestations les plus enflammées de la part des partisans du «linguistic turn». Non pas Stedman Jones, qui préfère ne pas intervenir sur les pages de Social History (déclaration implicite d'extériorité ${ }^{14}$, mais Patrick Joyce, le véritable protagoniste de l'affaire, et surtout James Vernon, dont le « Who's afraid of the linguistic turn » représente un effort abouti de synthèse des arguments postmodernistes ${ }^{15}$.

La référence à des recherches empiriques est considérée par tous ces auteurs comme le signe d'une impardonnable naïveté et est assimilée à la re-proposition d'un niveau « réel » et objectif, préexistant aux langages. Il faut poser le problème en d'autres termes : il s'agit d'arriver à une "conceptualisation du "social" comme construction discursive", dans laquelle les classes seraient essentiellement vues comme des constructions politiques. Il ne s'agit pas, avec cela, de nier la matérialité de la pauvreté ou de la richesse, «mais de soutenir qu'elles ne peuvent être comprises qu'à travers le langage [...]; nous ne pouvons avoir connaissance du réel ou de la société au-delà des discours ${ }^{16} »$. Et, du moment que, en outre, le réel peut seulement être saisi à travers nos catégories culturelles, laquelle de ses versions prévaudra ${ }^{17}$ ? Vernon, en particulier, se propose d'exposer la structure conceptuelle différente que les postmodernistes opposent à l'ancienne version représentée par Mayfield et Thorne et par Lawrence et Taylor. En travaillant sur ce que H. White a appelé le « contenu de la forme », il devient possible

[de] travailler au cœur de l'inconscient politique, d'interpréter les formes narratives comme des actes sociaux symboliques visant à réaliser l'aspiration humaine for a sense of agency. En suivant cette ligne, c'est la façon dont le discours social est construit comme une histoire dans laquelle les événements (réels ou imaginaires) sont dotés d'une signification et d'une cohérence dont ils seraient 
autrement dépourvus, qui permet aux sujets de donner un sens moral au monde et de s'imaginer eux-mêmes comme des agents à l'intérieur de celui-ci. De cette façon, on ne nie pas l'agency, au contraire. Une histoire sociale de ce genre est une histoire existentielle, une histoire du comment nous nous racontons comme agents unifiés. L'histoire de la différence est aussi une histoire de l'unité18. étant un authentique manifeste, on y trouve réunis efficacement nombre de points exposés sur le front postmoderniste du débat, principalement par Joyce. Surtout, ce que le texte aide finalement à tirer au clair est la nouvelle place assignée aux concepts-clés de l'historiographie sociale sur laquelle porte tout le débat: agency, expérience individuelle, expérience collective, conscience. L'« histoire existentielle » de Vernon est une histoire sans sujets individuels; ou mieux, une histoire où le sujet individuel se raconte comme unifié ; une histoire où la différence se transforme en unité. À la base de la construction des identités, les conditions matérielles ont été remplacées par les discours. La notion d'expérience individuelle est dissoute.

Il s'agit d'une approche qui doit beaucoup à une réflexion ponctuelle sur la question, développée quelques années auparavant par Joan Scott ${ }^{19}$. Le point de départ de J, Scott était lui aussi une critique de la vision structuraliste et déterministe de l'expérience implicite dans beaucoup de travaux des historiens sociaux, parmi lesquels ceux de E.P. Thompson (traduction subjective de la condition matérielle objectivement vécue et partagée par les membres d'un groupe; lien entre structure et conscience sociales ${ }^{20}$ ). Une vision radicalement différente lui était opposée, qui insistait sur le caractère discursif de l'expérience, sur ces discours qui positionnent les sujets et produisent leur expérience. «Ce ne sont pas les individus qui ont des expériences, mais les sujets qui se constituent à travers l'expérience ${ }^{21}$. " Le devoir de l'historien est de reconstruire les étapes du processus discursif dans lequel les identités sont inscrites. Cela implique un choix particulier d'objet: non pas les individus, mais «l'histoire de l'émergence des concepts et des identités comme des événements historiques qui demandent une explication ». Analyser des identités de race ou de sexe signifiera concevoir, au moins en partie, la position du sujet « comme effet des discours » sur la catégorie à laquelle il appartient ${ }^{22}$. Scott soutient que, malgré tout cela, ses sujets ont la capacité d'agir. Quand bien même ils seraient " constitués discursivement ", et l'expérience serait " un événement linguistique ", il reste toutefois un espace pour des modalités subjectives d'interprétation. Où ? Ce n'est pas clair. Ni même quel est l'objectif de cette précision, du moment que, réaffirme-t-elle, "par définition, le discours est partagé, et l'expérience est à la fois collective et individuelle ${ }^{23}$ ».

16 Résumons hypothèses et résultats des positions exprimées par Scott et Vernon. Point de départ, donc, la critique de la naturalisation du concept d'expérience et son usage sans scrupule par nombre d'historiens sociaux qui en ont fait un raccourci pour construire un sujet collectif (classe, groupe, mouvement...) sans s'interroger sur son existence en un temps et un lieu spécifiques. Point d'arrivée, la prise en compte de la nature discursive de l'expérience, qui permet de construire une histoire unifiée, où l'individuel et le collectif se confondent, tout comme se confondent différences et ressemblances.

17 [5] Je ne voudrais pas m'arrêter maintenant sur les implications relativistes de ces approches: non pas parce que je ne les considère pas comme significatives (tout au contraire ${ }^{24}$ ), mais parce que je voudrais que ces notes restent le plus possible liées au débat spécifique dont on parle. 
18 Il existe donc une forte convergence d'objectifs entre deux méthodes présentées comme antagonistes: la création de "groupes ", c'est-à-dire d'objets collectifs d'analyse. Ceci non pas à partir du constat de « ressemblance » ou du partage d'identité, mais à travers l'intervention de systèmes extérieurs aux acteurs sociaux, qui sont dotés d'extraordinaires capacités d'organisation (l'Économie ou la Culture). Dans les deux cas, l'historien réussit à fabriquer son objet favori d'analyse : le groupe, bien plus maniable que les individus fuyants ${ }^{25}$. Il faut bien dire que la référence de Vernon à l'histoire existentielle est un peu paradoxale. Le sujet unifié de Vernon, de Joyce et de Scott bégaye quelques paroles éparses (sa propre expérience), qui ne trouvent sens qu'à l'intérieur d'un système discursif capable de l'organiser ${ }^{26}$.

19 Était-il inévitable qu'une réflexion sur le concept d'expérience prenne cette voie ? Il me semble que non, surtout en regardant d'autres parcours suivis en particulier par de prétendus pères de l'approche postmoderniste. Que l'anthropologie herméneutique de Clifford Geertz diffère de l'application qui en est faite par de nombreux " geertzistes ", Giovanni Levi nous en avait déjà avertis il y a longtemps ${ }^{27}$. Une preuve irréfutable nous en est donnée par la lecture d'un livre consacré au thème de l'expérience, qui se termine par un "Épilogue » de C. Geertz ${ }^{28}$. Son titre, Anthropologie de l'expérience, nous expliquent ses directeurs, a été choisi parmi d'autres qui auraient pourtant fidèlement correspondu à leurs intentions et à son contenu : "Processual anthropology ", " Poststructural anthropology », "Interprétative anthropology ». Quoique partant de points de vue différents (Geertz, c'est connu, a lui-même explicité la distance entre sa méthode " textuelle » et celle "théâtrale » de Turner ${ }^{29}$ ), un accord s'est créé entre ces chercheurs sur le nécessaire dépassement d'une vision structuralo-fonctionaliste (c'està-dire naturaliste et déterministe) de l'expérience. Le point de départ de la discussion qui a réuni les collaborateurs du volume a été la réflexion philosophique de Wilhelm Dilthey ${ }^{30}$ et, surtout, la distinction entre expérience et comportement. Alors que la notion de comportement pourrait simplement renvoyer à une routine suivie par des individus, l'expérience fait référence à un être actif, qui n'est pas seulement impliqué dans une action, mais qui est capable de la charpenter.

Comment est-il possible de saisir l'expérience d'un acteur social? Il s'agit d'en interpréter les expressions. Entre l'expérience et ses expressions (c'est-à-dire, selon Dilthey, des actions, des représentations, des discours), les rapports sont à l'évidence circulaires, parce que l'expérience structure ses expressions, mais aussi parce que l'inverse est également vrai. Pour Dilthey, toutefois, cette relation dialogique et cette dépendance mutuelle ne sont pas embarrassantes, au contraire : le travail du chercheur se situe très exactement là, il se concentre sur l'analyse de ce rapport constant, non linéaire. Un des directeurs du volume, Bruner, fait à ce propos quelques observations que, paradoxalement, on croirait adressées aux postmodernistes (en polémique formelle contre toute forme de positivisme). Seul un positiviste aveugle, soutient Bruner, pourrait croire que les expressions correspondent à la réalité : tous les jours nous butons sur l'évidence du clivage qui existe entre les expériences et leurs manifestations symboliques. L'écart entre réalité, expérience et expressions est exactement l'objet d'étude de ces chercheurs. «Dans cette perspective, une expression ne pourra jamais être considérée comme un texte statique et isolé. Au contraire, elle implique une activité processuelle, une forme verbale, une action enracinée dans une situation réelle, avec des personnes réelles dans une culture particulière en un moment historique donné ${ }^{31}$.» L'expression est donc une forme historique, locale, fortement 
rattachée au sujet. Et en fait, selon Dilthey, «notre connaissance de ce qui constitue une expérience passe à travers l'interprétation de ses objectivations; et leur interprétation, d'autre part, est possible seulement si elle descend dans la profondeur de l'expérience subjective ${ }^{32}$ ».

21 Contre l'assimilation proposée par les postmodernistes entre expérience et discours, et la dépossession du sujet de sa propre expérience au profit du discours unitaire (dont la référence à la réalité est alternativement niée ou prise comme une donnée non problématisée), L'Anthropologie de l'expérience propose des analyses attentives à l'articulation entre ces différents plans : entre la réalité, l'expérience (la façon dont la réalité se présente à la conscience), les expressions (la façon dont l'expérience individuelle est structurée et articulée). L'échelle d'analyse est unique : « la profondeur de l'expérience subjective» capable de permettre d'analyser le processus de construction des comportements collectifs. Dans son épilogue, C. Geertz résume ce qu'il considère comme le devoir essentiel du chercheur : répondre à la question posée par un philosophe du XVIII siècle : «Comment est-il possible que nous partions tous comme des Originaux et que nous arrivions comme autant de Copies $^{33}$ ? " Le problème de l'articulation entre expérience individuelle et expérience collective est une donnée de départ, un moteur de la recherche: pour les postmodernistes, la construction du collectif est une assertion qui dérive de la centralité des discours. Geertz (du moins ce Geertz-là) n'est pas leur vrai père.

[6] C'est une perspective de ce genre, une perspective processuelle, qui est rappelée par Lawrence et Taylor. Une interrogation sur les identités collectives, prétendaient-ils, au lieu d'être un abandon de l'histoire sociale, exige d'affiner ses questions, de multiplier les sources utilisées et les points de vue à reconstruire. Ce que Vernon et Joyce ont taxé de positivisme ingénu était essentiellement une ébauche de réflexion (pas très approfondie, il est vrai) sur la nature des sources que l'historien trouve, utilise, crée en les rapprochant ou en les croisant. Comme il est prévisible, toute référence aux sources est absente de la réflexion postmoderniste. La réduction du document à un texte gouverné par des règles internes, par négation de tout renvoi à une réalité qui lui est extérieure, rend à l'évidence superflue toute réflexion sur ce thème. C'est bien d'ailleurs cette absence des sources qui rend le débat anglais un peu moins exotique que ce qu'il pourrait apparaître à première vue : parler de sources, reconstruire leur processus de formation, réfléchir sur les contraintes et les possibilités qu'elles offrent, ce sont là des opérations de plus en plus rares, même parmi les historiens continentaux, jusque parmi les auteurs qui se sont explicitement posé le problème de repenser les fondements du travail historique et les bases de la constitution d'une " communauté des historiens ${ }^{34}$ ».

23 Au-delà du texte de Lawrence et Taylor, le problème n'a été affronté, à ma connaissance, qu'une seule autre fois, sur les marges et à l'extérieur du cœur du débat, mais dans un texte très important. Dans le n 35 (1993) du History Workshop Journal, l'une des lettres adressées à la direction de la revue est signée E. P. Thompson. Dans cette lettre, finalement, on discute de sources, de contextes, de preuves.

24 La lettre de Thompson est une réaction indignée et, en même temps, affligée, à un article paru dans la même revue et écrit par l'un de ses pères fondateurs, Ralph Samuel, élève et proche depuis toujours de Thompson ${ }^{35}$. "Reading the Signs " avait été pensé comme une extraordinaire entreprise de médiation. Samuel s'était donné comme objectif d'inscrire le déconstructionisme dans la longue chaîne généalogique des 
travaux qui, depuis le début des années soixante, s'étaient détachés du positivisme plat et avaient manifesté un intérêt particulier pour la culture et ses représentations. L'attention aux «signes" devient ainsi le fil conducteur d'une relecture de l'historiographie des dernières décennies. À mon sens, la tentative n'est pas réussie. Il est difficile de ne pas être pris de vertige lorsque l'on voit, énumérés parmi les anticipateurs du déconstructionisme, Natalie Davis, Philippe Ariès (défini comme déconstructioniste avant la lettre), Carlo Ginzburg, Umberto Eco ou Piero Camporesi (dont les méthodes d'analyse seraient en partie débitrices de l'idéalisme de $\mathrm{Croce}^{36}$ ); il est également difficile de ne pas sursauter face à la confusion entre anthropologie interprétative et ethnométhodologie, micro-histoire et déconstruction ${ }^{37}$. Mais revenons à Thompson. L'article contenait aussi une réflexion plus ponctuelle sur les problèmes posés par le choix des contextes d'analyse - choix « crucial pour établir (ou suggérer) le "sens" que l'on veut offrir au lecteur d'un épisode unique ${ }^{38}$ ». Samuel prenait ici comme exemple, pour montrer le caractère totalement subjectif d'un tel choix, une série de travaux, dont Le grand massacre des chats de R. Darnton, et l'article sur les «ventes d'épouses " de Thompson ${ }^{39}$. Pour lui, l'interprétation par Thompson de ces marchés d'épouses dans l'Angleterre du XVIII ${ }^{e}$ siècle comme des formes de divorce informel était plausible; mais il aurait également été plausible d'interpréter ces épisodes dans le contexte de la violence domestique, ou dans celui de la traite des femmes, etc.

Dans la réponse de Thompson, le ton est à l'indignation : le choix du contexte d'analyse n'est pas dicté par le hasard ou l'humeur du chercheur. Quelle preuve pourrait soutenir l'affirmation d'un lien entre la vente des épouses et le contexte de la violence conjugale ou du marché des femmes? Preuve, possible, plausible, ce ne sont pas des termes interchangeables ${ }^{40}$. Le travail de l'historien est fait d'un dialogue constant entre questions (d'ordre théorique) et preuves. C'est là que se situent la liberté et les contraintes de l'historien ${ }^{41}$. La lettre s'achevait par quelques considérations affligées :

Le subjectivisme et l'idéalisme qui transparaissent dans le texte de Ralph Samuel sont aujourd'hui à la mode et nous devrons sans doute vivre avec durant les vingt prochaines années. Mais quelle sera la position de History Workshop Journal ? Quelle tristesse si Ralph Samuel suivait cette voie! Ce serait triste et inutile, du moment que le choix que l'on nous propose souvent entre un positivisme grossier et un idéalisme « plausible » est absolument faux.

Il me semble difficile de résumer plus efficacement l'équivoque dont nous sommes en train de débattre.

[7] E.P. Thompson: c'est ce nom, et ce qu'il représente, qui explique une des particularités de ce débat, c'est-à-dire l'extraordinaire violence du ton avec lequel les protagonistes s'apostrophent et qualifient les positions des adversaires. Le débat est "dépressif», il prend des accents "macho" et "phallocentriques"; on se bat pour revendiquer en réalité des orthodoxies faussement libérales et, au contraire, profondément "anti-libérales et réactionnaires "; la défense à outrance de l'histoire sociale exprime un mélange d'« apathie, insularité, défiance traditionnelle envers les théories continentales ${ }^{42} »$; on dénonce le fait d'être devenu le bouc émissaire des angoisses et des rages des autres ${ }^{43}$; d'autres font appel au contrôle des nerfs et des accusations ${ }^{44}$.

P. Joyce a sans aucun doute raison quand il soutient que le ton est difficilement séparable des contenus, dans un débat qui implique un contexte intellectuel «fortement politisé et moralisé ${ }^{45}$ ». Ce qui est en jeu est à l'évidence un héritage culturel, qui s'identifie largement avec l'œuvre de Thompson. C'est son nom qui revient 
de façon obsessionnelle dans tous les articles, comme mesure de fidélité ou de trahison. Une grande partie des protagonistes du débat sont ou ont été identifiés comme ses élèves ; beaucoup, qui ont actuellement pris leur distance, ont participé dans les années soixante-dix aux mêmes entreprises culturelles (et peut-être aussi politiques ${ }^{46}$ ). Tout cela a fait de ce groupe une génération, au-delà des éventuelles différences d'état-civil. Voilà qui explique le caractère quasi viscéral du débat.

En France, par exemple, la critique des catégories et des méthodes de l'histoire sociale largement pratiquée dans les années soixante et soixante-dix est moins passée par des affrontements directs; elle a davantage pris la forme de recherches parallèles en divers domaines (histoire culturelle, sociologie, etc.). S'identifiant largement à un conflit entre des générations différentes, cette critique a emprunté la voie du dépassement des limites évidentes du positivisme myope qui imprégnait une certaine histoire sociale (suscitant ainsi un débat interne plus qu'un affrontement; le dépassement est une conduite juvénile légitimée). Mais en Angleterre, ainsi qu'aux États-Unis, les choses sont différentes : comme les fractures sont aussi intergénérationnelles, elles se sont exprimées dans le langage violent qui caractérise les rapports à l'intérieur des groupes de pairs.

Les enjeux du débat interne au tournant linguistique en Angleterre sont donc de poids : il s'agit de définir légitimités, cohérences et trahisons. Quel dommage que ceux qui revendiquent la fidélité à l'œuvre de Thompson soient restés sourds à ses dernières propositions : sources, contextes, ces questions n'arrivent pas à entrer dans le débat qui reste avant tout idéologique, plus encore que théorique. C'est dommage, parce que Thompson avait profondément raison de dire qu'il n'est pas vrai que nous nous trouvions face à une alternative entre un positivisme grossier et un idéalisme plausible.

\section{NOTES}

1. L'ensemble des articles examinés ont paru dans Social History, entre 1992 et 1996 : D. Mayfield et S. Thorne, «Social History and its Discontents: Gareth Stedman Jones and the Politics of Language ", XVII, 1992, p. 165-188; J. Lawrence et M. Taylor, "The Poverty of Protest : Gareth Stedman Jones and the Politics of Language - a Reply", XVIII, 1993, p. 3-15; D. Mayfield et S. Thorne, "Reply to "The Poverty of Protest" and "The Imaginary Discontents" ", XVIII, 1993, p. 221-249 ; J. Vernon, «Who's Afraid of the Linguistic Turn? The Politics of Social History and its Discontents », XIX, 1994, p. 83-97 ; R. Gray, "Class, Politics and Historical "Revisionism" ", XIX, 1994, p. 209-220 ; N. Kirk, " History, Language, Ideas and Post-modernism : a Materialist View », XIX, 1994, p. 221-240 ; P. Joyce, "The End of Social History?», XX, 1995, p. 81-91; G. Eley et K. Nield, "Starting over : the Present, the Postmodern and the Moment of Social History ", XX, 1995, p. 355-364 ; P. Joyce, "The End of Social History? A Brief Reply to Eley and Nield », XXI, 1996, p. 96-98; M. W. Steinberg, «Culturally Speaking: Finding a Common between Poststructuralism and the Thompsonian Perspective ", XXI, 1996, p. 193-214.

2. Languages of Class. Studies in English Working Class History, 1832-1982, Cambridge, Cambridge University Press, 1983. 
3. Outcast London. A Study in the Relationship between Classes in Victorian Society, Oxford, Oxford University Press, 1971 ( $2^{\mathrm{e}}$ éd., Londres, 1975).

4. E. P. Thompson, La formation de la classe ouvrière anglaise [1963], Paris, Gallimard-Seuil, 1988.

5. Une première version de l'article était parue dans J. Epstein et D. Thompson, eds, The Chartist Experience. Studies in Working Class Radicalism and Culture, 1830-1860, Londres, Macmillan, 1982.

6. Languages of Class..., op. cit., p. 19-20.

7. Ibid., p. 21-22.

8. En particulier, R. Gray, «The Deconstructing of the English Working Class ", Social History, XI, 1986, p. 363-373 ; P. Schöettler, « Historians and Discourse Analysis », History Workshop Journal, 27, 1989, p. 37-65.

9. D. Mayfield et S. Thorne, « Social History and its Discontents », op. cit., p. 179-180.

10. J. Lawrence et M. Taylor, « The Poverty of Protest ", op. cit., p. 8.

11. Et plus encore le récent travail de P. Joyce, Democratic Subjects. The Self and the Social in Nineteenth-Century England, Cambridge, Cambridge University Press, 1994 ; pour les comptes rendus de l'ouvrage, cf. la note 15.

12. M. Taylor, "The linguistic Turns in British Social History », Bollettino del Diciannovesimo Secolo, 4, 1995, p. 5-13 (je dois cette référence à Bob Lumley). Taylor souligne justement que, en réalité, l'historiographie anglaise est aux prises avec au moins trois tournants linguistiques: celui «culturaliste» de E.P. Thompson, celui «contextualiste» de G. Stedman Jones, celui «postmoderniste» de P. Joyce. Cf. aussi l'article récent de M. Gervasoni, «Storia del lavoro e storia del linguaggio », Storica, 5, 1996, p. 115-128.

13. J. Lawrence et M. Taylor, «The Poverty of Protest », op. cit., p. 13.

14. Alors qu'il propose un bilan (pessimiste) de ces études dans "The Determinist Fix. Some Obstacles to the Further Development of the Linguistic Approach to History in the 1990's ", History Workshop Journal, 42, 1996, p. 19-35.

15. J. Vernon est l'auteur de Politics and the People. A Study in English Political Culture, c. 1815-1867, Cambridge, Cambridge University Press, 1993 ; deux utiles comptes rendus : D. Wahrman, «The New Political History: a Review Essay ", Social History, XXI, 1996, p. 343-353 (compte rendu de Vernon, Finn, Epstein, Joyce) ; R. Price, «Languages of Revisionism: Historians and Popular Politics in Nineteenth-Century Britain ", Journal of Social History, 30, 1996, p. 229-249 (compte rendu de Vernon, Biagini et Reid, M. Taylor, Joyce).

16. J. Vernon, « Who'Afraid... », op. cit., p. 89, 96.

17. P. Joyce, « The End... », op. cit., p. 78.

18. J. Vernon, « Who'Afraid... », op. cit., p. 93.

19. J. Scott, «The Evidence of Experience », Critical Inquiry, 17, 1991, p. 773-797. Pour une critique argumentée de ces positions, cf. L. Downs, «If "Women" is an Empty Category, why am I Afraid to Walk Alone at Night? Identity Politics meets the Postmodern Subject», Comparative Studies in Society and History (New York-Cambridge), XXXV, 1993, p. 414-451 (suivi d'une discussion avec J. Scott).

20. Cf. aussi W. H. Sewell, «How Classes are made. Critical Reflexions on the E. P. Thompson's Theory of Working Class Formation », in H. J. Kay et K. McClelland, eds, E. P. Thompson: Critical Perspectives, Cambridge, Polity Press, 1990, p.50-77; S. Cerutti, «Processus et Expérience. Individus, groupes et identités à Turin au XVIII siècle", in J. Revel, ed., Jeux d'échelles. La microanalyse à l'expérience, Paris, Gallimard-Seuil, 1996, p. 161-186, dans lequel la critique de Thompson suit toutefois une direction très différente de celle de J. Scott.

21. J. Scott, «The Evidence of Experience », op. cit., p. 779.

22. Ibid., p. 792.

23. Ibid., p. 793. 
24. Durant ces dernières années, elles ont été analysées en profondeur, dans de nombreux travaux, par Carlo Ginzburg, dont «Unus Testis. Lo sterminio degli Ebrei e il principio di realtà », Quaderni storici, 80, 1992, p. 529-548.

25. Cf. S. Cerutti, op. cit.

26. Sur l'annulation du sujet pratiquée par cette historiographie, les réflexions les plus approfondies restent celles de G. Levi, «I pericoli del geertzismo", Quaderni storici, 58, 1985, p. 269-277. Cf. en outre M. Sonenscher, Work and Wages. Natural Law, Politics and the EighteenthCentury French Trades, Cambridge, Cambridge University Press, 1989, p. 7-42.

27. G. Levi, op. cit.

28. V. Turner et E. Bruner, eds, The Anthropology of Experience. With an Epilogue by C. Geertz, Urbana, University of Illinois Press, 1986.

29. C. Geertz, «Genres flous : la refiguration de la pensée sociale", in Savoir local, savoir global. Les lieux du savoir, Paris, Presses universitaires de France, 1986, p. 27-47 (éd. américaine, New York, Basic Books, 1983).

30. Récemment repris par F. Ankersmit, "Can We Experience the Past ? , in R. Torstendhal et I. Velt-Brousse, eds, History Making. The Intellectual and Social Formation of a Discipline, Konferenser 37, Proceedings of an International Symposium, Uppsala, September 1994, Stockholm, 1996, p. 47-76.

31. V. Turner et E. Bruner, eds, The Anthropology of Experience..., op. cit., p. 6-7.

32. Ibid., p. 6.

33. Ibid., p. 380.

34. Cf. en ce sens G. Noiriel, Sur la « crise » de l'histoire, Paris, Belin, 1996. Font exception J. Boutier et D. Julia, eds, Passés recomposés. Champs et chantiers de l'Histoire, Paris, Autrement, 1995, et E. Artifoni et A. Torre, eds, Erudizione e fonti, Quaderni storici, 93, 1996.

35. R. Samuel, «Reading the Signs », History Workshop Journal, 32, 1991, p. 88-109; dans le $n^{\circ} 33$ (1992), paraît la suite de l'article, «Reading the Signs : II. Fact-grubbers and mind-readers ", p. 220-251, auquel Thompson se réfère.

36. R. Samuel, op. cit., p. 98.

37. R. Samuel, op. cit., passim, mais en particulier p. $102 \mathrm{sq}$.

38. R. Samuel, op. cit., II, p. 243.

39. R. Darnton, Le grand massacre des chats, Paris, Laffont, 1985 (éd. américaine New York, Basic Books, 1984) ; E. P. Thompson, Customs in Common, Londres, Merlin Press, 1991 (2 éd. Londres, Penguin, 1993, p. 404).

40. Cf. C. Ginzburg, «Prove e possibilità. In margine a il ritorno di Martin Guerre di Natalie Zemon Davis ", postface à N. Z. Davis, Il ritorno di Martin Guerre, Turin, Einaudi, 1984.

41. Arnaldo Momigliano avait répondu de la même façon aux arguments de Hayden White, «La retorica della storia e la storia della retorica : sui tropi di Hayden White », in Sui fondamenti della storia antica, Turin, Einaudi, 1984, p. 465-476.

42. J. Vernon, « Who's Afraid... », op. cit.

43. P. Joyce, « The End of Social History? A brief Reply », op. cit.

44. G. Eley et K. Nield, « Starting over », op. cit.

45. P. Joyce, « The End of Social History? A brief reply », op. cit., p. 96.

46. Les oppositions dont nous parlons ont des origines en réalité lointaines, qui remontent probablement à l'histoire de la New Left Review, dans les années soixante. La question mériterait d'être étudiée pour elle-même. 


\section{RÉSUMÉS}

L'article rend compte d'un débat historiographique, autour du "tournant linguistique », qui divise la communauté des historiens anglo-saxons. Il analyse les arguments avancés par ses partisans et ses détracteurs dans un lieu spécifique, la revue anglaise Social History, qui accueille depuis 1992 des échanges réguliers, parfois violents. Il précise les diverses positions, montrant comment, sous l'étiquette de " tournant linguistique ", se mélangent indûment des positions très souvent différentiées; il indique qui sont, outre-atlantique, les inspirateurs des historiens qui se réclament de ce "tournant linguistique », et sur quoi débouchent leurs analyses. Il s'agit alors d'interpréter les raisons de quelques importantes «censures" dans un débat qui ambitionne de rediscuter méthodes, finalités et objectif du travail de l'historien, en particulier l'absence de toute réflexion sur les sources et les contextes d'analyse (ce qui le rend moins exotique par rapport aux discussions historiographiques françaises). Enfin, l'article cherche à rendre compte de la violence inhabituelle du ton des affrontements, qui renvoie aux problèmes de l'héritage culturel de E. P. Thompson.

Notes on a Debate and its Censures. The paper reviews a historiographical debate around the "linguistic turn", which divides the community of anglo-saxon historians. It analyses the arguments put forward by its partisans and detractors in the specific space of the British journal Social History, which since 1992 has welcomed regular, sometimes even violent exchanges. It specifies the different positions, showing how under the label of "linguistic turn" very often differentiated positions are unduly mixed ; it indicates who are the inspirers of historians beyond the Atlantic claiming kinship with this "linguistic turn", and the outcome of their analyses. The question in such a case is to interpret the reasons for some important "censures" in a debate which aspires to re-discuss methods, finalities and objectives of the work of the historian, particularly the absence of all reflection on sources and the contexts of analysis (which renders it less exotic in relationship to French historiographical discussions). Finally, the paper seeks to account for the unusual violence of the style of oppositions, which refers to the problems of E.P. Thompson's cultural heritage.

\section{AUTEURS}

\section{SIMONA CERUTTI}

Simona Cerutti (EHESS) est l'auteur de La ville et les métiers. Naissance d'un langage corporatif (Turin, $\mathrm{XVII}^{e}$-XVIII ${ }^{e}$ siècle), Paris, Editions de l'EHESS, 1991, et de nombreux essais sur l'histoire sociale des sociétés urbaines d'Ancien Régime. Ses recherches portent actuellement sur le fonctionnement des tribunaux urbains au XVIII ${ }^{\mathrm{e}}$ siècle, sur la procédure judiciaire et les modalités de règlement des conflits. Elle est co-directeur de la revue Quaderni Storici. 